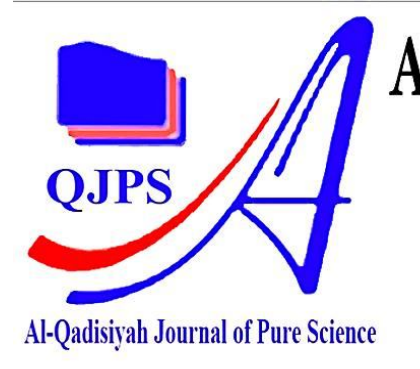

Al-Qadisiyah Journal of Pure Science

ISSN(Printed): 1997-2490

ISSN(Online):

$2411-3514$

DOI : /10.29350/jops.

http:// qu.edu.iq/journalsc/index.php/JOPS

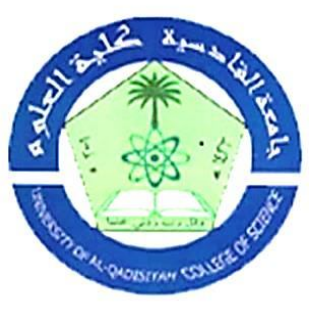

\title{
Stochastic Model of the Absorption of Drugs Problem in the Cells and Organs
}

\begin{tabular}{|c|c|}
\hline Authors Names & ABSTRACT \\
\hline $\begin{array}{l}\text { a. Sajjad Abd-AL Hussein } \\
\text { Haddad ALkha } \\
\text { b. Ihsan Jabbar Kadhim }\end{array}$ & $\begin{array}{l}\text { We proposed and analyzed the stochastic differential equation for the } \\
\text { problem of the absorption of drugs problem in the cells and organs with }\end{array}$ \\
\hline Article History & fixed volume. Firstly we studied the conditions of the local stable and \\
\hline $\begin{array}{l}\text { Received on: 18/12/ } 2020 \\
\text { Revised on: } 11 / 01 / 2021 \\
\text { Accepted on: } 14 / 01 / 2021\end{array}$ & $\begin{array}{l}\text { existence of all its equilibria are given. Finally numerical simulations of } \\
\text { various set of values of parameters are provided to confirm the }\end{array}$ \\
\hline $\begin{array}{l}\text { Keywords: } \\
\text { Stochastic differential } \\
\text { equation, Random dynamical } \\
\text { system, Ito integral, } \\
\text { Absorption of Drugs }\end{array}$ & $\begin{array}{l}\text { theoretical findings. The absorption of drugs is the One of the four } \\
\text { phases of pharmacokinetic movement, which includes absorption, } \\
\text { distribution, metabolism and excretion, abbreviated as (ADME). }\end{array}$ \\
\hline $\begin{array}{l}\text { DOI: https://doi.org/10.29350/ } \\
\text { jops.2021.26. 1.1255 }\end{array}$ & \\
\hline
\end{tabular}

\section{Introduction}

For mathematical analysis in biology it is often convenient to study and research the organisms (such as humans, animals and plants) as a group of single compounds called (compartments). The scientist Teorrell (1937) was the owner of the principle of pharmacokinetics, and he was the first to use the term (compartment) on cells and organs and divided them into (compartments), including one compartment and multi-compartment. The compartment may be an organ (such as the stomach, pancreas and liver) or a group of cells working together complement group. There is an important issue includes how to determine the mechanism of absorbing chemicals in living cells and organs, such as a drug. This issue has a practical application in the medicine's field, because it is possible that certain harmful drugs accumulate in an organ or in a group of cells, that ultimately damages them. The simplest issue of this kind is only about one compartment. It will also be useful for some purpose if we 
are interested in system that include two or more compartments (multi-compartments) that interact with each other. As expected, the difficulty of mathematical analysis increases when the number of compartments increase.

In this paper we shall use the Ito integral see [1],[3],[8] and [10] to solve the stochastic differential equation that represent our problem.

\section{Absorption of Drugs Problem in the Cells and Organs)}

In this section, we show that how can the problem of Absorption of Drugs in the Cells and Organs is modeled as a stochastic differential equation. Also, we solve this equation by using Ito integral. We have studied the linear pharmacokinetic movement of a single-compartment system.

\subsection{Pharmacokinetic Models in The Body:}

(a) Linear model of pharmacokinetics: The kinetics of the drug does not change with the change in the dose administered, the total clearance of the body from the drug is constant and the speed of transitions or reactions taking place are of the first order and there is a linear relationship between the given dose and the area of the surface under the curve . linear model of pharmacokinetics includes: One - compartment linear model, Two - compartment linear model and Multi-compartment linear model.

\section{(b) Nonlinear Model of Pharmacokinetics}

Pharmacokinetics changes with the change in the dose given to the patient, as the total clearance of the body from the drug is not constant, unlike in the case of linear mobility. In this model, the speed of transitions is zero and there is no linear relationship between the dose used and the surface area under the curve and this applies to the drugs that are metabolized by the body are mainly metabolized when given in high doses (toxicity). This model has special equations and special methods for calculating the kinematic factors of drugs that follow their kinetics. 


\section{Mathematical Model}

\subsection{One Compartment Model}

In this section we will study this type of pharmacokinetics model to create a stochastic model to describe drug absorption in this model This model describes the body as a single mass that has a certain size of distribution (V) related to several factors specific to the patient, such as age, health condition remains constant, and each drug has a certain apparent distribution size (Vd). In this model, the drug can be present immediately in the compartment after being given intravenously or gradually when given orally. It is assumed here that the drug is distributed immediately in the tissues (the entire compartment) and directly the equilibrium is done.

Consider carries drug into organs has volume $V \mathrm{~cm}^{3}$ with rate $a \mathrm{~cm}^{3} / \mathrm{sec}$ and out from the organs with rate $b \mathrm{~cm}^{3} / \mathrm{sec}$. We create a stochastic differential equation that describes the drug in the organs. The following diagram describes the single cell with volume $V$ and one inlet and one outlet

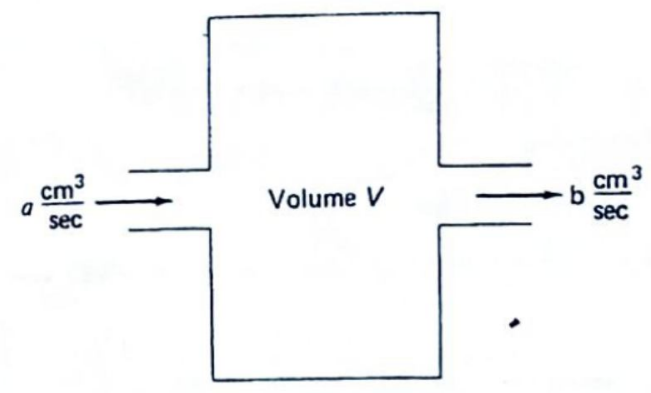

Let $X$ represented to the concentration of drug in the organs $\left(\mathrm{g} / \mathrm{cm}^{3}\right)$. Thus the quantity of the drug is given by

$$
\left(V \mathrm{~cm}^{3}\right)\left(X \mathrm{~g} / \mathrm{cm}^{3}\right)=V X g
$$

Here we consider the volume $V$ is constant and the concentration of drug depending on the time $t$. In general the concentration of drug is not deterministic.

That is it might happen that $X(t)$ is not completely known, but subject to some random ecological belongings, so the concentration of drug is

$$
X(t)+\text { "noise", }
$$

where we do not know the careful behavior of the noise term, only its probability distribution. Suppose that the function $X(t)$ is not random. Assume that the concentration of drug is $X(t)+\alpha W(t), W(t)=$ white noise, $\alpha=$ constant. Hence the quantity of the drug is given by $V(X(t)+\alpha W(t))$. Then the average rate of the quantity of the drug through the time $t$ is 


$$
\frac{d}{d t}(V(X(t)+\alpha W(t)))=V\left(\frac{d}{d t} X(t)+\alpha \frac{d}{d t} W(t)\right)
$$

The number of grams of the drug that is enter in the organ at the time $t$ is given by

$$
\left(a \frac{c m^{3}}{s e c}\right)\left(c \frac{g}{c m^{3}}\right)=a c \frac{g}{s e c}
$$

The number of grams of the drug that is ooze from the organ at the time $t$ is given by

$$
\left(b \frac{c m^{3}}{s e c}\right)\left(x \frac{g}{c m^{3}}\right)=b X \frac{g}{s e c}
$$

Now the average rate of the quantity of the drug equal to average of the entering drug in the organ minus the average of the oozing drug. So, from (3), (4) and (5) we have

$$
V\left(\frac{d}{d t} X(t)+\alpha \frac{d}{d t} W(t)\right)=a c-b X(t)
$$

Then

$$
\frac{d}{d t} X(t)=\left(\frac{a c}{V}-\frac{b}{V} X(t)\right)-\alpha \frac{d}{d t} W(t)
$$

or

$$
d X(t)=\left(\frac{a c}{V}-\frac{b}{V} X(t)\right) d t-\alpha d W(t)
$$

Assume that the concentration of drug at $t=0$ is $X=X_{0}$. So

$$
X=X_{0} \quad \text { whenever } t=0
$$

The solution of the intieal value problems given in (7) and (8) is given by

$$
\begin{gathered}
X(t)=e^{-\frac{b t}{V}}\left(X_{0}+\frac{a c}{V} \int_{0}^{t} e^{\frac{b s}{V}} d s\right)+\alpha \int_{0}^{t} e^{-\frac{b(t-s)}{V}} d W(s) \\
=e^{-\frac{b t}{V}} X_{0}+\frac{a c}{b}\left(1-e^{-\frac{b t}{V}}\right)+\alpha \int_{0}^{t} e^{-\frac{b(t-s)}{V}} d W(s) \\
=\frac{a c}{b}+e^{-\frac{b t}{V}}\left(x_{0}-\frac{a c}{b}\right)+\alpha \int_{0}^{t} e^{-\frac{b(t-s)}{V}} d W(s)
\end{gathered}
$$

Thus 


$$
X(t, \omega)=\frac{a c}{b}+e^{-\frac{b t}{V}}\left(X_{0}-\frac{a c}{b}\right)+\alpha \int_{0}^{t} e^{-\frac{b(t-s)}{V}} d W(s, \omega)
$$

is a solution of equation (7) with initial condition (8).

\subsection{Two Special Cases:}

Here we shall point to two cases that are important in many applications.

Case I: When $a=b$. In this case average of the entering drug in the organ equal to the average of the oozing drug. Hence

$$
X(t, \omega)=c+e^{-\frac{b t}{V}}\left(X_{0}-c\right)+\alpha \int_{0}^{t} e^{-\frac{b(t-s)}{V}} d W(s, \omega)
$$

Case II: $a=b$ and $X_{0}=0$. In this case average of the entering drug in the organ equal to the average of the oozing drug and the initial value of concentration of drug is zero. Whence

$$
X(t, \omega)=c\left(1-e^{-\frac{b t}{V}}\right)+\alpha \int_{0}^{t} e^{-\frac{b(t-s)}{V}} d W(s, \omega)
$$

The following proposition shows the asymptotical behavior of the solution of the Absorption of Drugs Problem that given equation (9).

Proposition 2.3: Consider the solution of the Absorption of Drugs Problem that given equation (9). Then we have

(i) $\lim _{t \rightarrow \infty} E[X(t, \omega)]=\frac{a c}{b}$.

(ii) $\lim _{t \rightarrow \infty} \operatorname{Var}[X(t, \omega)]=\frac{\alpha^{2} V}{2 b}$

Proof (i) The expected value of the solution $X(t, \omega)$ is given by

$$
\begin{aligned}
E[X(t, \omega)] & =E\left[\frac{a c}{b}+e^{-\frac{b t}{V}}\left(X_{0}-\frac{a c}{b}\right)\right]+\alpha E\left[\int_{0}^{t} e^{-\frac{b(t-s)}{V}} d W(s, \omega)\right] \\
& =\frac{a c}{b}+E\left[e^{-\frac{b t}{V}} X_{0}\right]-\frac{a c}{b} e^{-\frac{b t}{V}} \\
& =e^{-\frac{b t}{V}} E\left[X_{0}\right]+\frac{a c}{b}\left(1-e^{-\frac{b t}{V}}\right)
\end{aligned}
$$

Therefore 


$$
\lim _{t \rightarrow \infty} E[X(t, \omega)]=\lim _{t \rightarrow \infty}\left\{e^{-\frac{b t}{V}} E\left[X_{0}\right]+\frac{a c}{b}\left(1-e^{-\frac{b t}{V}}\right)\right\}=\frac{a c}{b}
$$

(ii) The variance of $X(t, \omega)$ is given by

$$
\begin{aligned}
\operatorname{Var}(X(t, \omega)) & =\operatorname{Var}\left(\frac{a c}{b}+e^{-\frac{b t}{V}}\left(X_{0}-\frac{a c}{b}\right)+\alpha \int_{0}^{t} e^{-\frac{b(t-s)}{V}} d W(s, \omega)\right) \\
& =e^{-2 \frac{b t}{V}} \operatorname{Var}\left(X_{0}\right)+\frac{\alpha^{2} V}{2 b}\left(1-e^{-2 \frac{b t}{V}}\right)
\end{aligned}
$$

So

$$
\lim _{t \rightarrow \infty} \operatorname{Var}[X(t, \omega)]=\lim _{t \rightarrow \infty}\left\{e^{-2 \frac{b t}{V}} \operatorname{Var}\left(X_{0}\right)+\frac{\alpha^{2} V}{2 b}\left(1-e^{-2 \frac{b t}{V}}\right)\right\}=\frac{\alpha^{2} V}{2 b}
$$

\section{Examples 2.4 (Computational Examples)}

(i) Let $V=600 \mathrm{~cm}^{3}, a=b=10 \mathrm{~cm}^{3} / \mathrm{sec}$ and $c=0.08 \mathrm{~g} / \mathrm{cm}^{3}$, also $(0, \omega)=0$. Then the concentration of drug after $30 \mathrm{sec}$ is given by

$$
X(t, \omega)=0.08\left(1-e^{-\frac{1}{2}}\right)+\alpha \int_{0}^{30} e^{-\frac{(30-s)}{60}} d W(s, \omega)
$$

the concentration of drug after $120 \mathrm{sec}$ is given by

$X(t, \omega)=0.08\left(1-e^{-2}\right)+\alpha \int_{0}^{120} e^{-\frac{(120-s)}{60}} d W(s, \omega)$

(ii) The concentration of the stability case is given by

$$
\lim _{t \rightarrow \infty} E[X(t, \omega)]=0.08 .
$$

(iii) The homogeneity of the concentration in the cell is given by

$$
\lim _{t \rightarrow \infty} \operatorname{Var}[X(t, \omega)]=30 \sigma^{2}
$$

Theorem 2.5 [10] Let $\mathcal{M}_{t}$ be a $\sigma$-field induced by $X_{s}$ for all $s \leq t$. Let $\mathcal{N}_{t}$ be a $\sigma-$ field induced by $\xi_{s}$ for all $s \leq t$ where $d \xi_{t}=\mu d t+\alpha d W(t), \xi_{0}=x$. Then

(i) $\mathcal{M}_{t}=\mathcal{N}_{t}$. 
(ii) $\tilde{B}_{t}=\int_{0}^{t} \alpha^{-1}\left(\mu-E\left[\mu / \mathcal{M}_{s}\right] d s+W_{t}\right.$ is a Brownian motion.

(iii) $d X_{t}(t)=E\left[\mu / \mathcal{M}_{t}\right] X_{t} d t+\alpha d \widetilde{W}_{t}$.

Corollary 2.6: Consider the stochastic differential equation (9)

$$
d X(t)=\left(\frac{a c}{V}-\frac{b}{V} X(t)\right) d t-\alpha d W(t)
$$

Then $X(t, \omega)$ can be represented as

$$
d X_{t}(t)=\left(E\left[\frac{a c}{V} / \mathcal{M}_{t}\right]-\frac{b}{V} X(t)\right) d t+\alpha d \widetilde{W}_{t}
$$

\section{Random Dynamical system}

Here we shall investigate the random dynamical system generated by (7). For the definition of random dynamical system, we refer to [3] and [6].

Theorem 3.1: the stochastic differential equation given by (7) generate a random dynamical system.

Proof Let $\theta$ be any metric dynamical system. Define $\varphi: \mathbb{R} \times \Omega \times \mathbb{R} \rightarrow \mathbb{R}$ defined by

$$
\varphi(t, \omega) x=\frac{a c}{b}+e^{-\frac{b t}{V}}\left(x-\frac{a c}{b}\right)+\alpha \int_{0}^{t} e^{-\frac{b(t-s)}{V}} d W(s, \omega)
$$

It easy to see that is $\varphi$ a cocycle over $\theta$. For

(i) It is clear that $\varphi(\cdot, \omega, \cdot): \mathbb{R} \times \mathbb{R} \rightarrow \mathbb{R}$ is continuous for every $\omega \in \Omega$. Also

$$
\varphi(t, ;, x): \Omega \rightarrow \mathbb{R} \text { is measurable for every }(t, x) \in \mathbb{R}^{2}
$$

(ii) $\varphi(0, \omega) x=\frac{a c}{b}+\left(x-\frac{a c}{b}\right)=x$.

(iii) $\varphi(t+r, \omega) x=\frac{a c}{b}+e^{-\frac{b(t+r)}{V}}\left(x-\frac{a c}{b}\right)+\alpha \int_{0}^{t+r} e^{-\frac{b(t+r-s)}{V}} d W(s, \omega)$

$$
\begin{aligned}
\varphi\left(t, \theta_{r} \omega, \varphi(r, \omega) x\right) & =\frac{a c}{b}+e^{-\frac{b t}{V}}\left(\varphi(r, \omega) x-\frac{a c}{b}\right)+\alpha \int_{0}^{t} e^{-\frac{b(t-s)}{V}} d W(s, \omega) \\
& =\frac{a c}{b}+e^{-\frac{b t}{V}} \varphi(r, \omega) x-e^{-\frac{b t}{V}} \frac{a c}{b}+\alpha \int_{0}^{t} e^{-\frac{b(t-s)}{V}} d W(s, \omega)
\end{aligned}
$$




$$
\begin{aligned}
& =\frac{a c}{b}+e^{-\frac{b t}{V}}\left(\frac{a c}{b}+e^{-\frac{b r}{V}}\left(x-\frac{a c}{b}\right)+\alpha \int_{0}^{r} e^{-\frac{b(r-s)}{V}} d W(s, \omega)-\frac{a c}{b}\right)+\alpha \int_{0}^{t} e^{-\frac{b(t-s)}{V}} d W(s, \omega) \\
& =\frac{a c}{b}+e^{-\frac{b t}{V}} \frac{a c}{b}+e^{-\frac{b t}{V}} e^{-\frac{b r}{V}}\left(x-\frac{a c}{b}\right)+\alpha e^{-\frac{b t}{V}} \int_{0}^{r} e^{-\frac{b(r-s)}{V}} d W(s, \omega)-e^{-\frac{b t}{V}} \frac{a c}{b} \\
& +\alpha \int_{0}^{t} e^{-\frac{b(t-s)}{V}} d W\left(s, \theta_{r} \omega\right) \\
& =\frac{a c}{b}+e^{-\frac{b(t+r)}{V}}\left(x-\frac{a c}{b}\right)+\alpha \int_{0}^{r} e^{-\frac{b(r+t-s)}{V}} d W(s, \omega) \\
& +\alpha \int_{0}^{t} e^{-\frac{b(t-s)}{V}} d W(s, \omega)
\end{aligned}
$$

Proposition 3.2: This RDS has an exponentially unstable $\mathcal{F}_{+}$-measurable equilibrium.

Proof: Since the factor $\frac{b}{V}>0$, then the random variable $u(\omega)$ defined by

$$
u(\omega)=\frac{a c}{b}-\frac{a c}{b} e^{\frac{b t}{V}}-\alpha \int_{0}^{\infty} e^{\frac{b s}{V}} d W(s, \omega)
$$

form the exponentially unstable $\mathcal{F}_{+}-$measurable equilibrium.

\section{Conclusion}

The stochastic model of the Absorption of Drugs in the Cells and Organs with fixed volume is coincide with the mean-reverting Ornstein- Uhlenbeck process. See [1], [9] and [10]. Also, from Proposition 2.2, model of the Absorption of Drugs is stable. If we set $\alpha=0$ in equation (3) we get the deterministic model of the Absorption of Drugs in the Cells. See Spiegel M.R., 1981.

\section{$R$ eferences}

[1] Allen E., 2007, Modeling with Itô Stochastic Differential Equations, Springer.

[2] Arnold L., 2003, Random dynamical systems, Springer, Berlin (Corrected 2nd printing).

[3] Arnold L., 2011, Random Dynamical Systems: Unfolding the Dynamics of Stochastic Differential Equations, Rome, Italy ENOC 2011, 24-29.

[4] Arnold L., 1995, M.Scheutzow, "Perfect Cocycles Through Stochastic Differential Equations". Probab. Th. Rel. Fields101: 65-88. 
[5] Baxendale P., 1984, "Brownian Motion in The Diffeomorphism Group I". Compositio Mathematica 53:19-50.

[6] Chueshov I., 2002, Monotone Random Systems Theory and Applications, Springer- Verlag Berlin Heidelberg Germany.

[7] Crauel H. and Gundlach M., 1999, Stochastic Dynamics, Springer-Verlag New York, Inc.

[8] Itô K., 1951, On Stochastic Differential Equations". Memoirs of the AMS, Volume 4.

[9] Mao X., 2010, Stochastic Differential Equations and Applications, $2^{\text {nd }}$ Edition, Woodhead Publishing Limited.

[10] Øksendal B., 2003, "Stochastic Differential Equations an Introduction with Applications", SpringerVerlag Berlin Heidelberg, $16^{\text {th }}$ ed.

[11] Spiegel M.R., 1981, Applied Differential Equations", Prentice Hall Inc. Englewood Cliff, New Jersey, Third Edition. 